\title{
How should an IACUC handle high mortality rates?
}

Madison Schute, who was responsible for training new members of her laboratory, walked slowly to her meeting with a subcommittee of the Great Eastern University IACUC. The issue was whether or not a new person in her laboratory was trained adequately to perform intracerebral (IC) injections in mice. During a regular semiannual inspection the IACUC inspectors found that almost half of all mice given intracerebral injections by the new lab member had died, whereas those administered by more experienced personnel were doing fine. Now Schute and the new research technician had to face an inquiry by the subcommittee.
Schute told the subcommittee that the new research technician was trained following her lab's standard IACUC approved procedures. That is, training began by the new person observing a skilled technician performing the technique. Then the new person, with Schute's oversight, practiced the technique on dead mice, progressing to doing the procedure with animals under non-recovery anesthesia, and finally to performing the injection under general anesthesia from which the animals recovered. In Schute's opinion the new technician was fully qualified to perform the procedure. The subcommittee thought otherwise, saying that the proof could be found in the unfortunate results of the injections when compared to the results from other lab members. Additionally, the subcommittee said the problem was compounded by Schute not advising the IACUC or any school veterinarian that there was a problem. Schute responded that she did not think that there was a problem because $30-50 \%$ mouse mortality was not unusual for a new person performing IC injections, and the IACUC protocol even listed extra animals to account for experimental failures.

Do you think that Schute's explanation is credible or does her lab have more of a problem than she realizes? How would you proceed with handling the finding from the semiannual inspection?

\section{RESPONSE}

\section{More oversight and communication might be needed}

Julie Fitzgerald, DVM, MS, DACVPM, CPIA, MAJ, VC

In our opinion, Schute has more of a problem than she realizes. Comparatively the new technician has a higher mortality rate than other technicians performing the same manipulation. This leads to the conclusion that the technician is not technically proficient. As stated in the Institutional Animal Care and Use Committee Guidebook, "training is a classic performance standard where the emphasis is on the outcome" . While Schute has assured the Great Eastern University IACUC that the employee was properly trained using IACUC approved methods, it is ultimately the responsibility of the IACUC to ensure that personnel conducting procedures on research animals are appropriately qualified and trained.

We recommend that the technician be suspended from performing the proce- dure in question until he or she can be retrained. As recommended in the Guide for the Care and Use of Laboratory Animals, training may need to be tailored to accommodate the educational background and experience of the individual ${ }^{2}$. Following laboratory standard operating procedures may not always be sufficient. If necropsies were performed to determine the exact cause of death of the mice, this could pinpoint the problem area that the retraining should focus on. Once retrained, this technician should continue to be closely monitored and the IACUC should request a periodic status update. Additionally, even though Schute is the training coordinator, she may have delegated the task of training to subordinate employees. The personnel that trained the new staff member should also be observed to make sure that proper techniques were taught.

Another point to make is that training and communication should never be the responsibility of one individual. The Great Eastern University animal care staff, including veterinarians and veterinary technicians, should also be actively involved in training at the University. We also feel that more open communication between Schute and the veterinary staff could have prevented some of the animal deaths. Once it was determined that there was an increase in the mortality rate for procedures performed by this technician, Schute should have addressed the issue with veterinary staff and temporarily excused the technician from performing the technique.

Schute's opinion that there is no problem is also cause for some concern. It may be a good idea for the IACUC to review Schute's qualifications and experience for this procedure, to ensure that she can adequately oversee training for her laboratory. Finally, the Great Eastern University IACUC should consider reviewing their post approval monitoring program, which could have identified this issue earlier.

1. ARENA/OLAW. Institutional Animal Care and Use Committee Guidebook 2nd edn. (OLAW, Bethesda, MD, 2002).

2. Public Health Service. Policy on Humane Care and Use of Laboratory Animals (US Department of Health and Human Services, Washington, DC, 1986, revised 2015).

Fitzgerald is Interim Chief, Department of Clinical Investigation and Chief, Bio Research Service, William Beaumont Army Medical Center, El Paso, TX. 